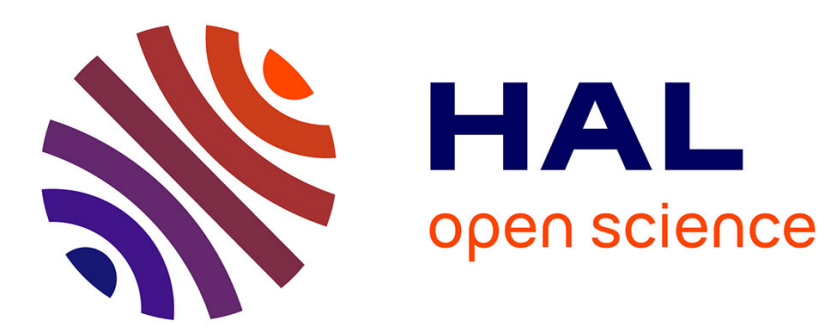

\title{
Spacecraft formation stabilization and fault tolerance: a state-varying switched system approach
}

Hao Yang, Bin Jiang, Vincent Cocquempot, Mou Chen

\section{To cite this version:}

Hao Yang, Bin Jiang, Vincent Cocquempot, Mou Chen. Spacecraft formation stabilization and fault tolerance: a state-varying switched system approach. Systems and Control Letters, 2013, 62 (9), pp.715-722. hal-00837519

\section{HAL Id: hal-00837519 \\ https://hal.science/hal-00837519}

Submitted on 22 Jun 2013

HAL is a multi-disciplinary open access archive for the deposit and dissemination of scientific research documents, whether they are published or not. The documents may come from teaching and research institutions in France or abroad, or from public or private research centers.
L'archive ouverte pluridisciplinaire HAL, est destinée au dépôt et à la diffusion de documents scientifiques de niveau recherche, publiés ou non, émanant des établissements d'enseignement et de recherche français ou étrangers, des laboratoires publics ou privés. 


\title{
Spacecraft formation stabilization and fault tolerance: a state-varying switched system approach *
}

\author{
Hao Yang ${ }^{a}{ }^{\dagger}$, Bin Jiang ${ }^{a}$, Vincent Cocquempot ${ }^{b}$, Mou Chen ${ }^{a}$ \\ ${ }^{a}$ College of Automation Engineering \\ Nanjing University of Aeronautics and Astronautics \\ 29 YuDao Street, Nanjing, 210016, China. \\ ${ }^{b}$ LAGIS, UMR CNRS 8219 \\ Université Lille 1 : Sciences et Technologies \\ 59655, Villeneuve d'Ascq cedex, France.
}

\begin{abstract}
The focus of this paper is the spacecraft formation flying problem where the formation switches successively among multiple shapes, the number and the composition of spacecraft in the group may change among these shapes, and some spacecraft may be faulty. The whole flying process is modeled as a state-varying switched system. The formation stability and fault tolerability are analyzed by using new results on state-varying switched systems.
\end{abstract}

Keywords: Spacecraft formation; Switched systems; State variation; Stabilization; Fault tolerant control.

\section{Introduction}

The development of a reliable, autonomous and highly accurate formation keeping strategy for multiple spacecraft is a prerequisite for future space missions such as large space interferometer or large synthetic aperture radar, etc [1]. The formation control problem of spacecraft has been a research topic for many years [2]-[8]. Existing techniques can be divided into three architectures [1]: 1) Leader-follower, where one spacecraft is regarded as the leader and is tracked by other following spacecraft; 2) Virtual structure, in which the spacecraft are treated as rigid bodies embedded in an overall virtual rigid body; 3) Behavioral, where several desired behaviors are prescribed for each spacecraft, whose individual controllers are designed to achieve these behaviors.

${ }^{*}$ This work is supported by National Natural Science Foundation of China $(61034005,61104116,61273171)$, Doctoral Fund of Ministry of Education of China (20113218110011), and a Project Funded by the Priority Academic Program Development (PAPD) of Jiangsu Higher Education Institutions.

${ }^{\dagger}$ Corresponding author: Tel: +86 25 84892301-6060, Fax: +86 25 84892300. Email: haoyang@nuaa.edu.cn (H. Yang), binjiang@nuaa.edu.cn (B. Jiang), vincent.cocquempot@univ-lille1.fr (V. Cocquempot), chenmou@nuaa.edu.cn (M. Chen) 
The formation shape for a group is not always fixed, which may change according to different mission requirements. Various optimal approaches have also been developed to achieve the formation reconfiguration from one shape to another one with considering the path planning, collision avoidance, fuel optimality, etc [9]-[10].

On the other hand, Fault-Tolerant Control (FTC) aims at retaining acceptable performance under the system faults [11]. FTC is highly required for spacecraft control systems. Formation flying performances would be seriously degraded due to actuators, sensors, controllers or internal components failures $[12,13,14]$. Only a few results are devoted to the FTC of spacecraft formation, we refer to [15] where a multiple level framework that comprehensively adjusts individual controllers of the faulty spacecraft and formation configurations is proposed.

In this work, we focus on a class of spacecraft formation flying processes with three behaviors: b1. the formation shape switches successively among several shapes; b2. the number and the composition of spacecraft in the group may change among different shapes due to four phenomena: docking, undocking, departure, and participation (formal definitions will be given later); b3. Some spacecraft may be faulty. The above three behaviors reveal important characteristics and the complexities of spacecraft formation flying process in the real situation. Although these three behaviors have been investigated respectively, few literatures consider b1, b2 and b3 simultaneously.

We model the whole flying process by a state-varying switched nonlinear system with each mode related to one shape and the states representing the formation errors of spacecraft in the group. This allows us to analyze the formation stability and fault tolerability by using switched system theory [17]-[21]. Unfortunately, most of existing results on switched systems deal with one single state space and are hard to apply to the state-varying case because the states of each mode are no longer the same as others.

The considered state variation covers two cases:

Case 1. some states in different modes are closely related;

Case 2. some states in different modes are completely independent.

For Case 1, Ref.[16] introduces a concatenation operator which represents the relations between states of different modes. Another similar idea is to consider a "large" state vector that can be used for all modes, and model the state variation by using reset maps (or impulsive effects) [22, 23]. These modeling methods however can not be applied to Case $\mathbf{2}$ since no relation can be established between independent states, the independent states' values of the mode to be activated at next switching time can not be determined by the states' values in the current mode.

In this work, we effectively analyze the effect of state variations resulting from $\mathbf{b} \mathbf{2}$ on the system. Our contributions are two-fold: 
1. We extend switched system stability results to the case of varying states, and propose a new formation stability criterion that relies on the switching law, the individual controllers of each spacecraft, and the trade-off among the values of the new spacecraft' formation errors when they just join the group.

2. We transfer the fault tolerance problem into a stability problem of switched system with unstable modes. According to state variation behaviors, we show a special way of fault tolerance that relies on switching law and does not reconfigure the individual controllers. This can be combined with other FTC approaches to make the FTC scheme more flexible.

The obtained results can not only be used to design spacecraft formation flying process a priori, but also to check the fault tolerability without control reconfiguration in real time.

In the rest of the paper: Section 2 addresses the modeling issue and gives the problem formulation. Section 3 discusses the formation keeping problem as well as the FTC strategy. Simulation results are shown in Section 4, followed by conclusions in Section 5 .

\section{Formation modeling and problem formulation}

In this section, we first model the specified spacecraft formation process by a state-varying switched system, then formulate the formation control problem.

\subsection{Interconnection architecture}

The formation configuration with its leader-following interconnection architecture are shown in Fig. 1. The group consists of a main spacecraft (square) and several subordinate ones (round). The main spacecraft has the capability of docking and undocking with subordinate ones. Each subordinate spacecraft may leave/join the group over the time. The main spacecraft is the leader of the group, each subordinate spacecraft is a follower which only receives the information from the main one.

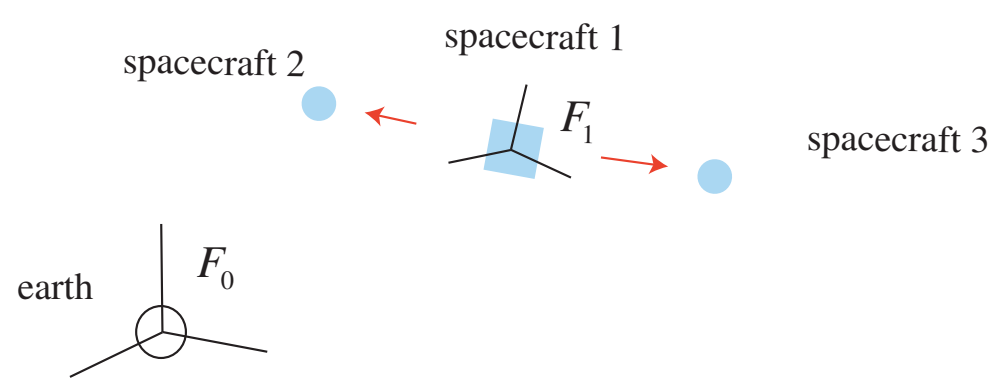

Figure 1: Interconnection architecture and coordinate frames 
Such architecture has two benefits 1) A main spacecraft needs to communicate with its subordinate ones to complete the docking/undocking. This can be achieved conveniently by the leaderfollowing structure. 2) Each subordinate spacecraft may be docked with the main one or leave/join the group over the time. The proposed architecture prevents each subordinate spacecraft from communicating with other subordinate ones. This makes the interconnection topology and formation control design simpler.

\subsection{Relative dynamics}

Consider a group of $n$ spacecraft, define a finite set $\mathcal{N} \triangleq\{1,2, \ldots, n\}$. Spacecraft 1 denotes the main spacecraft, and spacecraft $i, i \in \mathcal{N}-\{1\}$ is the $i$ th subordinate one. $F_{0}$ denotes an inertial reference frame fixed at the center of the earth; $F_{i}$ denotes a body-fixed reference frame located at the mass center of spacecraft $i, i \in \mathcal{N} ; A_{i}$ denotes the arbitrary vector measured w.r.t. the origin of $F_{i} . \overrightarrow{A_{i}}$ denotes the related vector of $A_{i}$ measured w.r.t. the origin of $F_{0}$.

The translational dynamics of spacecraft $i(i \in \mathcal{N})$ is given by [25]:

$$
\left\{\begin{array}{l}
{\overrightarrow{R_{i}}}^{\prime}=\vec{V}_{i} \\
M_{i}{\overrightarrow{V_{i}}}^{\prime}=\overrightarrow{f_{e i}}+\overrightarrow{f_{i}}+\overrightarrow{d_{i}} \quad i \in \mathcal{N}
\end{array}\right.
$$

where $M_{i}$ denotes the mass of spacecraft $i, \vec{R}_{i}$ and $\vec{V}_{i}$ denote the position and velocity of its mass center, $\overrightarrow{f_{e i}} \triangleq-\frac{\mu M_{i}}{\left|\overrightarrow{R_{i}}\right|^{3}} \vec{R}_{i}$ represents the inverse-square gravitational force, where $|\cdot|$ is the Euclidean norm, $\mu \triangleq M g$ with $M$ being the mass of the earth and $g$ the universal gravitational constant, $\overrightarrow{f_{i}}$ is the control force, $\overrightarrow{d_{i}}$ describes uncertainties including disturbances and noise. It is assumed that $\left|d_{i}\right| \leq \bar{d}$ for a known constant $\bar{d}>0$. The symbol ' denotes the time derivative of the vector measured in $F_{0}$.

Define $\tilde{R}_{i} \triangleq C_{i}^{0}\left(\overrightarrow{R_{i}}-\overrightarrow{R_{1}}\right)$ and $\tilde{V}_{i} \triangleq C_{i}^{0}\left(\overrightarrow{V_{i}}-\overrightarrow{V_{1}}\right)$, where $C_{a}^{b}$ is the rotational matrix (its expression will be given later) that transforms the components of a vector expressed in $F_{b}$ to the components of the same vector expressed in $F_{a}$. Denote $\tilde{R}_{i}^{d}$ and $\tilde{V}_{i}^{d}$ as the desired relative position and velocity. Define $e_{R_{i}} \triangleq \tilde{R}_{i}^{d}-\tilde{R}_{i}, e_{V_{i}} \triangleq \tilde{V}_{i}^{d}-\tilde{V}_{i}$.

The relative translational dynamics between spacecraft $i(i \in \mathcal{N}-\{1\})$ and 1 in frame $F_{i}$ can be further obtained [8]:

$$
\left\{\begin{array}{l}
\dot{e}_{R_{i}}=e_{V_{i}}-\omega_{i}^{\times} e_{R_{i}} \\
\dot{e}_{V_{i}}=-\omega_{i}^{\times} e_{V_{i}}+\underbrace{\dot{\tilde{V}}_{i}^{d}+\omega_{i}^{\times} \tilde{V}_{i}^{d}-C_{i}^{1} \vec{V}_{i}^{\prime}+\frac{\mu}{\left|\vec{R}_{i}\right|^{3}} R_{i}}_{\Phi_{i}}-\frac{1}{M_{i}} f_{i}-\frac{1}{M_{i}} d_{i}
\end{array}\right.
$$

where $\omega_{i}$ represents the angular velocity of $F_{i}$, the symbol ${ }^{\circ}$ denotes the time derivative of the vector 
measured in $F_{i}$. The cross product of a vector $a=\left[\begin{array}{lll}a_{1} & a_{2} & a_{3}\end{array}\right]^{\top}$ is defined as:

$$
a^{\times} \triangleq\left[\begin{array}{ccc}
0 & -a_{3} & a_{2} \\
a_{3} & 0 & -a_{1} \\
-a_{2} & a_{1} & 0
\end{array}\right]
$$

The attitude dynamics of spacecraft $i(i \in \mathcal{N})$ in frame $F_{i}$ is given by (see [25]):

$$
\left\{\begin{array}{l}
J_{i} \dot{\omega}_{i}=-\omega_{i}^{\times} J_{i} \omega_{i}+\tau_{g i}+\tau_{i}+\iota_{i} \\
\dot{q}_{i}=\frac{1}{2}\left(q_{4 i} \omega_{i}-\omega_{i}^{\times} q_{i}\right) \\
\dot{q}_{4 i}=-\frac{1}{2} \omega_{i}^{\top} q_{i}
\end{array}\right.
$$

where $q_{i} \in \Re^{3}$ and $q_{4 i} \in \Re$ denote the quaternions, $J_{i}=J_{i}^{\top}$ is the positive definite inertia matrix, $\tau_{g i} \triangleq \frac{3 \mu}{\left|\overrightarrow{R_{i}}\right|^{5}} R_{i}^{\times} J_{i} R_{i}$ denotes the gravity gradient torque, $\tau_{i}$ is the control torque, $\iota_{i}$ describes uncertainties, $\left|\iota_{i}\right| \leq \bar{\iota}$ for a known constraint $\bar{\iota} \geq 0$.

Define $\tilde{\omega}_{i} \triangleq \omega_{i}-C_{i}^{1} \omega_{1}, \tilde{q}_{i} \triangleq q_{i}-C_{i}^{1} q_{1}$, and $\tilde{q}_{4 i} \triangleq q_{4 i}-C_{i}^{1} q_{4 j}$. Denote $\tilde{\omega}_{i}^{d}$, $\tilde{q}_{i}^{d}$ and $\tilde{q}_{4 i}^{d}$ as the desired relative angular velocity and quaternions. Define $e_{\omega_{i}} \triangleq \tilde{\omega}_{i}^{d}-\tilde{\omega}_{i}, e_{q_{i}} \triangleq \tilde{q}_{i}^{d}-\tilde{q}_{i}, e_{q_{4 i}} \triangleq \tilde{q}_{4 i}^{d}-\tilde{q}_{4 i}$.

The relative attitude dynamics between spacecraft $i(i \in \mathcal{N}-\{1\})$ and 1 can be obtained [8]:

$$
\left\{\begin{array}{l}
J_{i} \dot{e}_{\omega_{i}}=\underbrace{-\omega_{i}^{\times} J_{i} \omega_{i}+\frac{3 \mu}{\left|\overrightarrow{R_{i}}\right|^{5}} R_{i}^{\times} J_{i} R_{i}-J_{i}\left(C_{i}^{0} \omega_{i}\right)^{\times} \tilde{\omega}_{i}+J_{i} \omega_{i}^{\times} \tilde{\omega}_{i}^{d}-J_{i} \dot{\tilde{\omega}}_{i}^{d}-J_{i} C_{i}^{1} J_{1}^{-1} J_{1} \dot{\omega}_{1}}_{\Upsilon_{i}}+\tau_{i}+\iota_{i} \\
\dot{e}_{q_{i}}=\frac{1}{2}\left(e_{q_{4 i}} e_{\omega_{i}}-e_{\omega_{i}}^{\times} e_{q_{i}}\right) \\
\dot{e}_{q_{4 i}}=-\frac{1}{2} e_{\omega_{i}}^{\top} e_{q_{i}}
\end{array}\right.
$$

where the rotational matrix is defined as $C_{a}^{b} \triangleq\left(q_{4 a}^{2}-q_{a}^{\top} q_{a}\right) I_{3}+2 q_{a} q_{a}^{\top}+2 q_{4 a} q_{a}^{\times}$.

The main spacecraft 1 follows the given reference dynamics taking the following form

$$
\left\{\begin{array}{l}
{\overrightarrow{R_{r}}}^{\prime}=\overrightarrow{V_{r}} \\
M_{r}{\overrightarrow{V_{r}}}^{\prime}=\overrightarrow{f_{e r}}+\overrightarrow{f_{r}} \\
J_{r} \dot{\omega}_{r}=-\omega_{r}^{\times} J_{r} \omega_{r}+\tau_{g r}+\tau_{r} \\
\dot{q}_{r}=\frac{1}{2}\left(q_{4 r} \omega_{r}+\omega_{r}^{\times} q_{r}\right) \\
\dot{q}_{4 r}=-\frac{1}{2} \omega_{r}^{\top} q_{r}
\end{array}\right.
$$

The relative dynamics of spacecraft 1 can also be obtained as (2) (with $1=r, i=1, d_{r}=0$ ) and (5) (with $1=r, i=1, \iota_{r}=0$ ).

Denote the state and the individual controller of spacecraft $i(i \in \mathcal{N})$ as

$$
x_{i} \triangleq \Re^{13}=\left[e_{R_{i}}^{\top} e_{V_{i}}^{\top} e_{\omega_{i}}^{\top} e_{q_{i}}^{\top} e_{q_{4 i}}\right]^{\top}, \quad u_{i} \in \Re^{6} \triangleq\left[\begin{array}{ll}
f_{i}^{\top} & \tau_{i}^{\top}
\end{array}\right]^{\top}
$$

Define $X \in \Re^{13 \times n} \triangleq\left[x_{1}^{\top}, \ldots x_{n}^{\top}\right]^{\top}, d \triangleq\left[d_{1}^{\top}, \ldots d_{n}^{\top}\right]^{\top}, \iota \triangleq\left[\iota_{1}^{\top}, \ldots \iota_{n}^{\top}\right]^{\top}$. Under the connection architecture in Section 2.1, we can write the relative translational and attitude dynamics of all spacecraft with some state-feedback controllers $u_{i}\left(x_{i}\right)[3]$ as

$$
\dot{X}=f(X, d, \iota)
$$

where the function $f$ is determined from (2), (5), and the form of $u_{i}\left(x_{i}\right)$. 


\subsection{Switched system model}

Suppose that in the whole flying process, the spacecraft formation switches among $m$ known and fixed shapes. Since there is a formation reconfiguration process from one fixed shape to another one, we regard each reconfiguration process as a varying transition shape. Suppose that there exist $p$ possible varying shapes in the sequence.

Define $\mathcal{M}=\{1,2, \ldots, m+p\}$. The flying process is modeled by a state varying switched system

$$
\dot{X}_{\sigma}=f_{\sigma}\left(X_{\sigma}, d_{\sigma}, l_{\sigma}\right)
$$

where $\sigma(t):[0, \infty) \rightarrow \mathcal{M}$ denotes the switching signal, which is assumed to be a piecewise constant function continuous from the right. Mode $j$ (when $\sigma=j$ ) represents the relative dynamics (8) in formation shape $j . X_{j} \in \Re^{13 \times n_{j}}$ with $n_{j}$ being the number of spacecraft in mode $j$.

In any desired fixed shape, the desired relative states $\tilde{R}_{i}^{d}, \tilde{V}_{i}^{d}, \tilde{\omega}_{i}^{d}, \tilde{q}_{i}^{d}$ and $\tilde{q}_{4 i}^{d}$ among spacecraft are prescribed and time-invariant, they are time-variant in varying shapes. All desired relative states are designed to be continuous throughout the whole process.

\subsection{State variation among formation shapes}

Now we formally describe the state variation among formation shapes. Suppose that at $t=t_{k}$, the formation switches from mode $i$ to mode $j$. Denote $x_{q}\left(t_{k}^{-}\right)$and $x_{q}\left(t_{k}\right)$ respectively the states of spacecraft $q$ in mode $i$ and mode $j$, where $t^{-}$denotes the left limit time instant of $t$. The states vary due to 4 reasons:

1) Docking. Several subordinate spacecraft (each has one docking port) move to a main spacecraft (which has multiple docking ports installed symmetrically ${ }^{1}$ ) and are docked with it to form a new main spacecraft. Note that all relative states are continuous. Therefore the states value of the new main spacecraft is the same as that of the previous main spacecraft. The docking rule is:

$$
x_{1}\left(t_{k}\right)=x_{1}\left(t_{k}^{-}\right)
$$

2) Undocking. The main spacecraft 1 in mode $i$ are separated from $s$ subordinate ones in mode $j$ with states $x_{\varrho+1}, \ldots x_{\varrho+s}, \varrho \geq 1$. It is natural that just after docking, the state value of each new spacecraft is the same as that of the original main one. The undocking rule is:

$$
x_{\varrho+v}\left(t_{k}\right)=x_{1}\left(t_{k}\right)=x_{1}\left(t_{k}^{-}\right), \text {for } 1 \leq v \leq s
$$

\footnotetext{
${ }^{1}$ This makes the spacecraft's mass distribution nearly symmetric about a certain axis before and after docking so as to reduce the burden of its attitude control system [24].
} 
3) Departure. Some subordinate spacecraft in mode $i$ depart from the group at $t_{k}$, they are not involved in the formation and do not affect the formation flying performance, therefore their states are not considered in mode $j$.

4) Participation. Some spacecraft that do not exist in mode $i$ join the group at $t_{k}$, they are involved in the formation flying after $t_{k}$, their states can be regarded as new states and are considered in mode $j$. The spacecraft that departed before may come back which is also considered as a new spacecraft.

Based on above four phenomena, a state variation model between modes $i$ and $j$ is given

$$
X_{j}\left(t_{k}\right)=\left[\left(S_{i j} X_{i}\left(t_{k}^{-}\right)\right)^{\top} X_{j i}^{\star \top}\left(t_{k}\right)\right]^{\top}
$$

where $S_{i j}$ is a $n_{j} \times n_{i}$ non-zero constant variation matrix. This excludes an unpractical situation where all spacecraft depart from the formation simultaneously (in this case, the formation flying is ended). Therefore $\left|S_{i j}\right| \geq 1$. $X_{j i}^{\star}$ denotes the new states of mode $j$ w.r.t. mode $i$.

\subsection{Problem formulation}

To this end, we provide the formation stability definition as follows:

Definition 1: The formation flying is said to be practically stable if for the corresponding switched system (9), there exist a switching function $\sigma(t)$ and individual controllers $u_{i}$ such that for any $\epsilon>0$, there exist $\delta>0$ and $\varsigma>0$ such that

$$
\left|X_{\sigma(0)}(0)\right| \leq \delta \Longrightarrow\left|X_{\sigma(t)}(t)\right| \leq \epsilon+\varsigma D, \quad \forall t \geq 0
$$

where $D \triangleq\left|[\bar{d} \bar{l}]^{\top}\right|$.

The control goal of this paper is to guarantee that (11) holds for any given $\epsilon$ in the healthy and some faulty situations. The disturbance bound $D$ is often much less than the magnitude of the desired relative states, thus the precision of formation flying can be guaranteed under Definition 1.

\section{Formation stabilization analysis}

\subsection{Formation control in the healthy situation}

A simple state-feedback controller $u_{i}, i \in \mathcal{N}$ originated from [25] is provided as

$$
\begin{aligned}
& f_{i}=M_{i}\left(\Phi_{i}+\left(K_{1}+1\right) e_{R_{i}}+K_{2} e_{V_{i}}\right) \\
& \tau_{i}=-\Upsilon_{i}-J_{i}\left(K_{3} e_{\omega_{i}}+e_{q_{i}}+\frac{e_{\omega_{i}}}{2\left(e_{\omega_{i}}^{\top} e_{\omega_{i}}+\kappa\right)}\left(K_{4} e_{q_{i}}^{\top} e_{q_{i}}+K_{5} e_{q_{4 i}}^{\top} e_{q_{4 i}}\right)\right)
\end{aligned}
$$


where $\Phi_{i}$ is defined in (2), $\Upsilon_{i}$ is defined in (5), $K_{1}, K_{2}, K_{3}, K_{4}, K_{5}$ are negative constants representing feedback gains, $\kappa>0$ is an arbitrarily small constant.

Choose a Lyapunov candidate $V_{i}=\frac{1}{2} x_{i}^{\top} x_{i}$ where $x_{i}$ is given in (7). The time derivative of $V_{i}$ along (2) and (5) under controllers (12) and (13) satisfies

$$
\dot{V}_{i}=-K_{1}\left|e_{R_{i}}\right|^{2}-K_{2}\left|e_{V_{i}}\right|^{2}-\frac{e_{V_{i}}^{\top} d_{i}}{M_{i}}-K_{3}\left|e_{\omega_{i}}\right|^{2}-K_{4}\left|e_{q_{i}}\right|^{2}-K_{5}\left|e_{q_{4 i}}\right|^{2}-e_{\omega_{i}}^{\top} e_{q_{i}}+e_{\omega_{i}}^{\top} \iota_{i}
$$

Pick three constants $\nu_{1}>0$ and $\nu_{2}>0$ such that

$$
\begin{gathered}
-\frac{e_{V_{i}}^{\top} d_{i}}{M_{i}} \leq \nu_{1}\left|e_{V_{i}}\right|^{2}+\frac{\left|d_{i}\right|^{2}}{\nu_{1} M_{i}^{2}} \\
e_{\omega_{i}}^{\top} \iota_{i} \leq \nu_{2}\left|e_{\omega_{i}}\right|^{2}+\frac{\left|\iota_{i}\right|^{2}}{\nu_{2}} \\
-e_{\omega_{i}}^{\top} e_{q_{i}} \leq \nu_{3}\left|e_{\omega_{i}}\right|^{2}+\frac{\left|e_{q_{i}}\right|^{2}}{\nu_{3}}
\end{gathered}
$$

Substituting (15)-(17) into (14) with selecting $K_{2}>\nu_{1}, K_{3}>\nu_{2}+\nu_{3}, K_{4}>\frac{1}{\nu_{3}}$ leads to

$$
\dot{V}_{i} \leq-\eta V_{i}+\varepsilon\left(\bar{d}^{2}+\bar{\iota}^{2}\right)
$$

where $\eta>0 \triangleq 2 \min \left[K_{1}, K_{2}-\nu_{1}, K_{3}-\nu_{2}-\nu_{3}, K_{4}-\frac{1}{\nu_{3}}, K_{5}\right], \varepsilon \triangleq \max _{i \in \mathcal{N}}\left[\frac{1}{\nu_{1} M_{i}^{2}}, \frac{1}{\nu_{2}}\right]$.

Define $W \triangleq \sum_{i=1}^{n} V_{i}$, note that $W$ can be written as $W=X^{\top} X$, one has from (18)

$$
\dot{W} \leq-\eta W+n \varepsilon D^{2}
$$

Note that $\kappa$ in (13) is a positive constant that can be chosen arbitrarily small. Inequality (18) holds except when $e_{\omega_{i}} \rightarrow 0$ (in this case $\tau_{i}=-\Upsilon_{i}-J_{i} K_{3} e_{\omega_{i}}-J_{i} e_{q_{i}}, \dot{V}_{i} \leq 0$ ). Due to the uncertainties, $e_{\omega_{i}}$ will finally only converge to a small neighboring region of origin with respect to $D$ and can not converge to zero. Therefore, inequality (18) always holds except when $e_{\omega_{i}} \rightarrow 0$ temporarily in some possible short periods that can be neglected in practical situations.

Remark 1: Various state-feedback control approaches besides (12)-(13) can be designed to achieve (19). In the absence of full state measurement (especially the acceleration measurements), observerbased controller can also be applied. In this case, the right side of inequality (19) may include the observation error terms. The detailed controller design is not the focus of this paper and thus is not discussed.

For the shape $j, j \in \mathcal{M}$, inequality (19) is rewritten as

$$
\dot{W} \leq-\eta W+n_{j} \varepsilon D^{2}, \quad j \in \mathcal{M}
$$

Define $\zeta \triangleq \max _{j \in \mathcal{M}} \sqrt{n_{j} \varepsilon}, S \triangleq \max _{i, j \in \mathcal{M}}\left|S_{i j}\right|$. 
For the switched system (9), denote by $t_{i}, i=1,2, \ldots$ the $i$ th switching instant, $t_{0}=0$. It follows that mode $\sigma\left(t_{i}\right)$ is activated in the interval $\left[t_{i}, t_{i+1}\right)$. It is required that $\inf _{i}\left(t_{i+1}-t_{i}\right) \geq \tau$ where $\tau>0$ is called "dwell-time" [17]. Let $t_{j k}, j \in \mathcal{M}, k=1,2, \ldots$ be the $k$ th time when mode $j$ is switched on.

Theorem 1: For any $\epsilon$, the formation flying can be stabilized by $\sigma$ and controllers (12)-(13) if

$$
\begin{aligned}
\Delta t & \geq \max \left[\frac{2}{\eta} \ln \left(\frac{\epsilon+(S+2) \zeta D}{\epsilon_{1}}\right), \tau\right] \\
\left|X_{i \sigma\left(t_{i k}^{-}\right)}^{\star}\left(t_{i k}\right)\right| & \leq \epsilon_{2}+\zeta D
\end{aligned}
$$

where $0 \leq \epsilon_{2}<\epsilon$ and $\epsilon_{1}>0$ such that $S \epsilon_{1}+\epsilon_{2} \leq \epsilon . \Delta t$ denotes the minimal activating time of all modes.

Before proving Theorem 1, we provide some insight into the conditions (21)-(22).

- Condition (21) reveals the relation between the decay rate (related to the individual controllers) and the activating time of each mode. Given a fixed $\epsilon$ and $\epsilon_{2}$, a large (small) $\eta$ allows for a short (long) $\Delta t$. This implies that for a group whose states converge fast (slow) under individual controllers in each formation shape, the minimal activating time of all shapes would be chosen short (long).

- For a state-varying switched system, not all states appear from the beginning, some states may also disappear in some modes and appear again in other modes. Condition (22) imposes an upper bound $\epsilon_{2}+\zeta D$ w.r.t uncertainties on the new states $X_{i \sigma\left(t_{i k}^{-}\right)}^{\star}$ when they appear at $t_{i k}$. Such bound is reasonable since the new arrival spacecraft will be considered as a member of the group only after it approaches its desired trajectories and fits into the formation shape. A bounded $X_{i \sigma\left(t_{i k}^{-}\right)}^{\star}\left(t_{i k}\right)$ can be achieved by the controllers of the new arrival spacecraft according to the prescribed switching formation shape. Note that a large (small) $\epsilon_{2}$ implies a small (large) $\epsilon_{1}$, combining (21) and (22) one finds that relaxing (restricting) the bound of $\left|X_{i \sigma\left(t_{i k}^{-}\right)}^{\star}\left(t_{i k}\right)\right|$ leads to restriction (relaxation) on activating time $\Delta t$.

Proof of Theorem 1: Consider the time $t \in\left[0, t_{1}\right)$. It follows from (20) that

$$
\begin{aligned}
\left|X_{\sigma(0)}(t)\right|^{2} & \leq e^{-\eta t}\left|X_{\sigma(0)}(0)\right|^{2}+\int_{0}^{t} e^{-\eta(t-s)} \zeta^{2} D^{2} d s \\
& \leq e^{-\eta t}\left|X_{\sigma(0)}(0)\right|^{2}+\zeta^{2} D^{2}
\end{aligned}
$$

One further has

$$
\left|X_{\sigma(0)}(t)\right| \leq e^{-\frac{\eta}{2} t}\left|X_{\sigma(0)}(0)\right|+\zeta D
$$

Choose initial states such that

$$
\left|X_{\sigma(0)}(0)\right| \leq \epsilon+(S+1) \zeta D
$$


Substituting (24) and (21) into (23) yields $\left|X_{\sigma(0)}(t)\right| \leq \epsilon+(S+2) \zeta D, \forall t \geq 0$, and $\left|X_{\sigma(0)}\left(t_{1}^{-}\right)\right| \leq$ $\epsilon_{1}+\zeta D$.

At switching instant $t_{1}$, according to state variation model (10) we further have

$$
\begin{aligned}
\left|X_{\sigma\left(t_{1}\right)}\left(t_{1}\right)\right|^{2} & =\left|S_{\sigma\left(t_{1}\right) \sigma(0)} X_{\sigma(0)}\left(t_{1}^{-}\right)\right|^{2}+\left|X_{\sigma\left(t_{1}\right) \sigma(0)}^{\star}\left(t_{1}\right)\right|^{2} \\
& \leq S^{2}\left|X_{\sigma(0)}\left(t_{1}^{-}\right)\right|^{2}+\left|X_{\sigma\left(t_{1}\right) \sigma(0)}^{\star}\left(t_{1}\right)\right|^{2} \\
& \leq\left(S\left|X_{\sigma(0)}\left(t_{1}^{-}\right)\right|+\left|X_{\sigma\left(t_{1}\right) \sigma(0)}^{\star}\left(t_{1}\right)\right|\right)^{2}
\end{aligned}
$$

It follows from condition (22) that

$$
\begin{aligned}
\left|X_{\sigma\left(t_{1}\right)}\left(t_{1}\right)\right| & \leq S\left|X_{\sigma(0)}\left(t_{1}^{-}\right)\right|+\left|X_{\sigma\left(t_{1}\right) \sigma(0)}^{\star}\left(t_{1}\right)\right| \\
& \leq S\left(\epsilon_{1}+\zeta D\right)+\epsilon_{2}+\zeta D \\
& \leq \epsilon+(S+1) \zeta D
\end{aligned}
$$

Now consider $t \in\left[t_{1}, t_{2}\right)$, similar to $(23)$, one has

$$
\left|X_{\sigma\left(t_{1}\right)}(t)\right| \leq e^{-\frac{\eta}{2}\left(t-t_{1}\right)}\left|X_{\sigma\left(t_{1}\right)}\left(t_{1}\right)\right|+\zeta D
$$

Substituting (26) and (21) into (27) yields $\left|X_{\sigma\left(t_{1}\right)}(t)\right| \leq \epsilon+(S+2) \zeta D, \forall t \geq t_{1}$, and $\left|X_{\sigma\left(t_{1}\right)}\left(t_{2}^{-}\right)\right| \leq$ $\epsilon_{1}+\zeta D$.

By induction, we can conclude that under conditions (21)-(22)

$$
\left|X_{\sigma(t)}(t)\right| \leq \epsilon+(S+2) \zeta D, \quad \forall t \geq 0
$$

This completes the proof.

\section{$3.2 \quad$ Fault tolerant formation control}

Fault tolerant control (FTC) methods have been widely investigated for spacecraft [11]-[15]. A general FTC idea is to reconfigure the individual controllers of each faulty spacecraft, such that it still plays its desired role in the formation flying. Such FTC strategy obviously takes time and control cost [11], and is limited for controller faults since it is hard to reconfigure the controller to accommodate the fault in itself. Therefore, it is meaningful to look for an economical and simple FTC solution without reconfiguring the individual controllers. It will be shown that such solution exists for state-varying switched systems under some conditions.

Consider the fault that may occur in each subordinate spacecraft's internal equipments, actuators or even controllers, and changes inequality (14) into

$$
\dot{V} \leq \eta_{1} V+\varepsilon\left(\bar{d}^{2}+\bar{\iota}^{2}\right), \quad \text { for } \eta_{1}, \varepsilon>0
$$


which is a general inequality describing the instability of the relative dynamics in the faulty case. This means that the faulty subordinate spacecraft may deviate from its desired translational and attitude dynamics, and can not follow the main spacecraft expectedly.

Consequently, inequalities (20) of the whole group becomes

$$
\dot{W} \leq \eta_{1} W+n_{j} \varepsilon D^{2}, \quad j \in \mathcal{M}
$$

What we are interested in is whether the FTC goal can be achieved by the switching law without reconfiguring any individual controller.

Consider two faulty cases:

Case 1. The faulty spacecraft will depart at next switching time.

Case 2. The faulty spacecraft will merge with a main spacecraft at next switching time.

Rewrite $X=\left[\left(X^{h}\right)^{\top},\left(X^{f}\right)^{\top}\right]$, where $X^{h}\left(X^{f}\right)$ is the state vector of the healthy (faulty) spacecraft. Divide $\Delta t=\Delta t_{1}+\Delta t_{2}$, where $\Delta t_{1}$ is the period when there is no fault, while in $\Delta t_{2}$ the system is faulty. We do not discuss the detailed fault diagnosis (FD) procedure, which can be done by various diagnosis methods [11]. However, the well-known fault diagnosis delay (Such delay, or a bound of it, can generally be estimated a priori) is considered and included in $\Delta t_{2}$.

Theorem 2: For any $\epsilon$, the formation flying in cases 1 and 2 can be stabilized by $\sigma$ without reconfiguring controllers (12)-(13) if (21)-(22) hold, and

$$
\frac{\eta}{2} \Delta t_{1}-\frac{\eta_{1}}{2} \Delta t_{2} \geq 0
$$

Proof: Suppose that a fault occurs in mode $\sigma\left(t_{i}\right)$ that is activated in $\left[t_{i}, t_{i+1}\right)$, denote $t_{f}$ as the time after which the fault appears, i.e., $\Delta t_{1}=t_{f}-t_{i}, \Delta t_{2}=t_{i+1}-t_{f}$.

We first consider the state behaviors of the healthy spacecraft. Under conditions (21)-(22), one has from the proof of Theorem 1 that

$$
\begin{aligned}
\left|X_{\sigma\left(t_{i}\right)}^{h}(t)\right| & \leq e^{-\frac{\eta}{2}\left(t-t_{i}\right)}\left|X_{\sigma\left(t_{i}\right)}^{h}\left(t_{i}\right)\right|+\zeta D \\
& \leq e^{-\frac{\eta}{2}\left(t-t_{i}\right)}(\epsilon+(S+1) \zeta D)+\zeta D, \quad \forall t \in\left[t_{i}, t_{i+1}\right)
\end{aligned}
$$

and $\left|X_{\sigma\left(t_{i}\right)}^{h}\left(t_{i+1}^{-}\right)\right| \leq \epsilon_{1}+\zeta D$.

Now consider the behavior of whole state vector that satisfies

$$
\begin{aligned}
& \left|X_{\sigma\left(t_{i}\right)}(t)\right| \leq e^{-\frac{\eta}{2}\left(t-t_{i}\right)}(\epsilon+(S+1) \zeta D)+\zeta D, \quad \forall t \in\left[t_{i}, t_{f}\right) \\
& \left|X_{\sigma\left(t_{i}\right)}(t)\right| \leq e^{\frac{\eta_{1}}{2}\left(t-t_{f}\right)}\left(e^{-\frac{\eta}{2} \Delta t_{1}}(\epsilon+(S+1) \zeta D)+\zeta D\right)+\frac{e^{\frac{\eta_{1}}{2}\left(t-t_{f}\right)}}{\sqrt{\eta_{1}}} \zeta D, \quad \forall t \in\left[t_{f}, t_{i+1}\right)
\end{aligned}
$$


Substituting (29) into (31) one obtains that

$$
\left|X_{\sigma\left(t_{i}\right)}(t)\right| \leq \epsilon+(S+1) \zeta D+e^{\frac{\eta_{1}}{2}\left(t-t_{f}\right)} \zeta D+\frac{e^{\frac{\eta_{1}}{2}\left(t-t_{f}\right)}}{\sqrt{\eta_{1}}} \zeta D, \quad \forall t \in\left[t_{i}, t_{i+1}\right)
$$

Since $\Delta t_{2}$ is bounded, it can be seen from (32) that formation stability is guaranteed in $\left[t_{i}, t_{i+1}\right)$

In both Case 1 and Case 2, once the switching occurs, $X_{\sigma\left(t_{i}\right)}^{f}\left(t_{i+1}^{-}\right)$disappears. Therefore

$$
\left.\left|X_{\sigma\left(t_{i}\right)}\left(t_{i+1}^{-}\right)\right|=\mid X_{\sigma\left(t_{i}\right)}^{h}\left(t_{i+1}^{-}\right)\right) \mid \leq \epsilon_{1}+\zeta D
$$

The rest of the proof follows the same way as in Theorem 1 .

The main idea behind Theorem 2 is to transfer the fault tolerance problem into the stability problem of switched system with unstable modes. Condition (29) implies that if the faulty period is not very long meaning that before the faulty spacecraft departs from the group or merges with a main spacecraft, then the norm of the whole states value is always bounded.

Theorem 2 provides a special FTC clue for formation flying that is to eliminate the effect of the faulty spacecraft on the formation stability by letting it leave the group. This idea can be combined with other FTC approaches that rely on control reconfiguration to make the FTC scheme more flexible in real time.

\subsection{Control architecture}

Fig. 2 shows the control architecture of each spacecraft. For spacecraft $i(i \in \mathcal{N})$, all possible relative dynamics together with its related individual controllers can be designed a priori off-line (based on previous analysis) and then saved and marked in supervisor $i$. The switching sequence and switching times are often prescribed a priori according to certain mission requirements. These information are also saved and marked in supervisor $i$. Supervisor $i$ is inherently a discrete event system under which spacecraft $i$ applies its individual controllers to achieve the switching formation flying. Once the fault occurs, the supervisor takes the information of the fault and determines whether the fault is tolerable by switching law (if it is not available, then control reconfiguration scheme will be applied).

It can be seen that the proposed architecture is distributed, each spacecraft only receives the information from its neighbors (in the considered formation of this paper, the main spacecraft is the unique neighbor of all subordinate spacecraft). Such architecture combines leader-following and behavioral approaches.

Remark 2: The proposed switched system model can be extended to the case that the leaderfollowing structure is applied among subordinate spacecraft. In this case, each subordinate spacecraft may have multiple neighbors, the state dimension of $x_{i}$ may be more than 13 . However, 


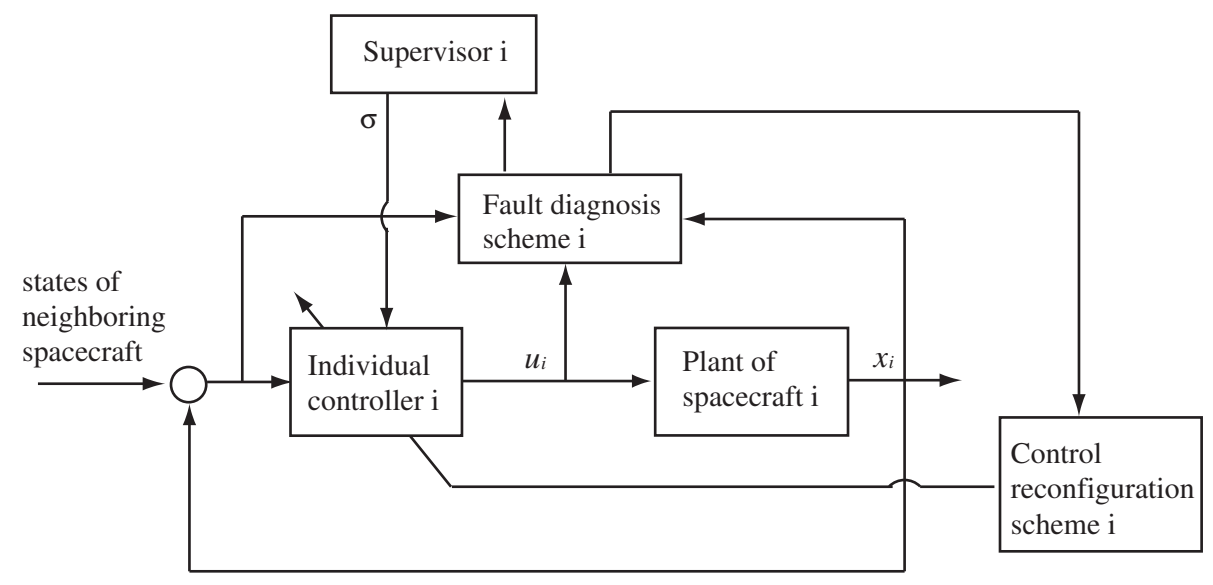

Figure 2: Control architecture for spacecraft $i$

once the reference subordinate spacecraft is docked with the main one or leaves the group, some spacecraft have to find another reference, their relative dynamics need to be recalculated, and their states are completely different from previous ones (the state transformation may be done by some geometrical methods). This makes the formation stabilization and fault tolerance analysis more complicated.

\section{Simulation results}

Consider a formation flying process with three desired and fixed shapes as shown in Fig. 3. In the transition from formation 1 to 2 , the main spacecraft 1 disassembles into main spacecraft 1 and subordinate spacecraft 4,5 . In the transition from formation 2 to 3 , spacecraft 4,5 are docked with main spacecraft 1 again; spacecraft 2 departs from the group and a new spacecraft 6 joins it in formation 3 . The whole process is modeled by a switched system with five modes (modes 1,3 , 5 are related to 3 desired shapes, while modes 2 and 4 represent varying transition shapes).

Denote $\left.\tilde{\vartheta}_{i}^{d} \triangleq\left[\tilde{q}_{i}^{d}\right)^{\top}, \tilde{q}_{4 i}^{d}\right]^{\top}, \tilde{x}_{i}^{d} \triangleq\left[\left(\tilde{R}_{i}^{d}\right)^{\top},\left(\tilde{V}_{i}^{d}\right)^{\top},\left(\tilde{\omega}_{i}^{d}\right)^{\top},\left(\tilde{\vartheta}_{i}^{d}\right)^{\top}\right]^{\top}$.

In mode $1, \tilde{R}_{2}^{d}=\left[\begin{array}{lll}0 & 500 & 0\end{array}\right]^{\top} m^{3}, \tilde{R}_{3}^{d}=\left[\begin{array}{lll}0 & -500 & 0\end{array}\right]^{\top} m^{3}, \tilde{V}_{2}^{d}=\tilde{V}_{3}^{d}=\left[\begin{array}{lll}0 & 0 & 0\end{array}\right]^{\top}(m / s)^{3}, \tilde{\omega}_{2}^{d}=$

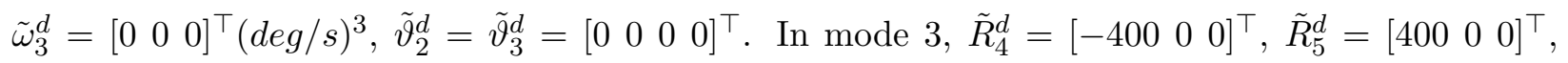

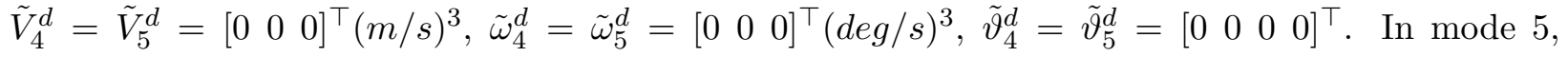

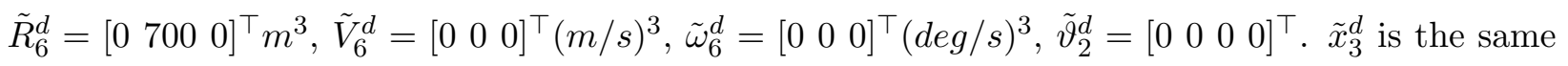
in modes $1-5, \tilde{x}_{2}^{d}$ is the same in modes $1-4$.

For two transition formations, in mode $2, \tilde{R}_{4}^{d}=\left[\int_{1000}^{2000} \tilde{V}_{4}^{d}(t) d t \quad 00\right]^{\top}, \tilde{R}_{5}^{d}=\left[\begin{array}{lll}\int_{1000}^{2000} \tilde{V}_{5}^{d}(t) d t & 0 & 0\end{array}\right]^{\top}$ with

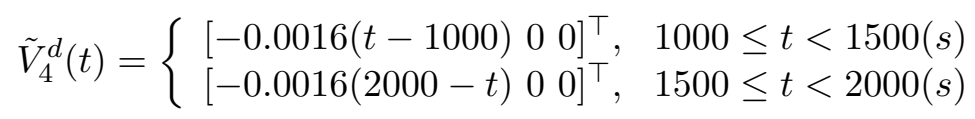




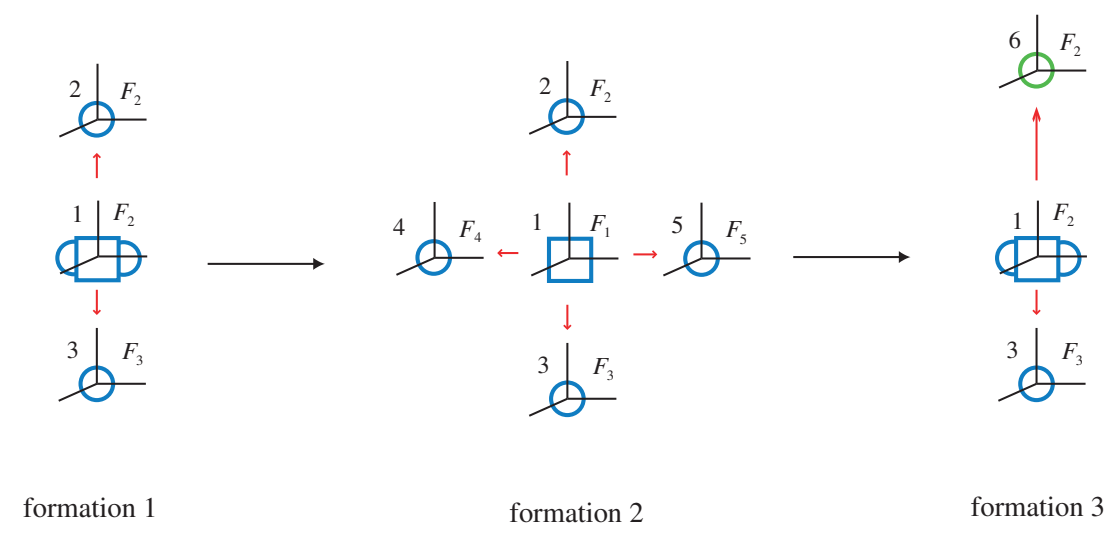

Figure 3: Spacecraft formation example

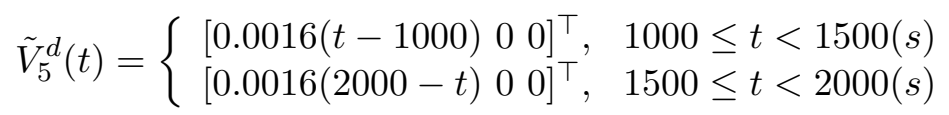

It can be seen that $\tilde{R}_{4}^{d}, \tilde{V}_{4}^{d}, \tilde{R}_{5}^{d}$ and $\tilde{V}_{5}^{d}$ are continuous throughout modes $1,2,3$. In mode 4 , these four variables are chosen similarly as in mode 2 .

$\tilde{x}_{1}^{d}=0$ for all modes. The reference dynamics which the main spacecraft 1 follows always satisfies $\overrightarrow{R_{r}}=\left[\begin{array}{lll}0 & 0 & 0\end{array}\right]^{\top} m^{3}, \overrightarrow{V_{r}}(t)=\left[\begin{array}{lll}0 & 0 & 0\end{array}\right]^{\top}(m / s)^{3}, \omega_{r}=\left[\begin{array}{lll}0 & 0 & 0\end{array}\right]^{\top}(\operatorname{deg} / s)^{3}, q_{r}=\left[\begin{array}{lll}0 & 0 & 0\end{array}\right]^{\top}, q_{4 r}=1$.

It is clear that the variation matrices are

$$
S_{12}=\left[\begin{array}{lll}
1 & 0 & 0 \\
0 & 1 & 0 \\
0 & 0 & 1 \\
1 & 0 & 0 \\
1 & 0 & 0
\end{array}\right], \quad S_{45}=\left[\begin{array}{lllll}
1 & 0 & 0 & 0 & 0 \\
0 & 0 & 0 & 0 & 0 \\
0 & 0 & 1 & 0 & 0
\end{array}\right]
$$

Therefore $S=\sqrt{5}$. For all spacecraft, $M_{i}=150 \mathrm{~kg} J_{i}=25 I_{3 \times 3} \mathrm{kgm}^{2} . M_{c}=1 \mathrm{~kg}, J_{c}=$ $I_{3 \times 3} \mathrm{kgm}^{2}$. The dwell-time is $\tau=80 \mathrm{~s}$. Appropriate design of individual controllers of each spacecraft yields $\zeta=5, \eta=0.01$. Suppose that $\bar{d}=0.06, \bar{\iota}=0.08$, this results in $D=0.1$. Choose $\epsilon=0.9, \epsilon_{1}=0.2, \epsilon_{2}=0.4$ which means that spacecraft 6 joins the formation after its state norm is no more than 0.9 .

We first consider the healthy situation. According to conditions (21)-(22), choose $\Delta t=1000 \mathrm{~s}$, the initial state norm is 1.5. Fig. 4 shows the trajectories of $X_{\sigma(t)}(t)$ where spacecraft 6 joins the formation at $t=4000 \mathrm{~s}$ when its state norm is 0.2 . We can see that $\left|X_{\sigma(t)}(t)\right|$ is always bounded within the prescribed region.

Now consider an individual controller fault of spacecraft 2 occurred in mode 4 , that is

$$
f_{i}=M_{i}\left(\Phi_{i}+\left(K_{1}+1\right) e_{R_{i}}+K_{2 f} e_{V_{i}}\right)
$$

which implies that in the control unit the channel that receives signals $e_{V_{i}}$ is faulty that makes the feedback gain $K_{2}$ change into $K_{2 f}$. Inequality (28) follows with $\eta_{1}=0.01$. Such phenomenon 
are commonly seen in the controller of the spacecraft that would be influenced by the radiation or magnetic field in the space environment. According to condition (29), the formation stability is still guaranteed if $\Delta t_{1} \geq \Delta t_{2}$. At $t=4000 \mathrm{~s}$, the departure of spacecraft 2 is completed, the formation switches to mode 5 . Suppose that the fault occurs at $t=3800 \mathrm{~s}$. Fig. 4 also shows that the stability is maintained even if $\left|x_{2}\right|$ increases in the interval $[3800,4000)(s)$.
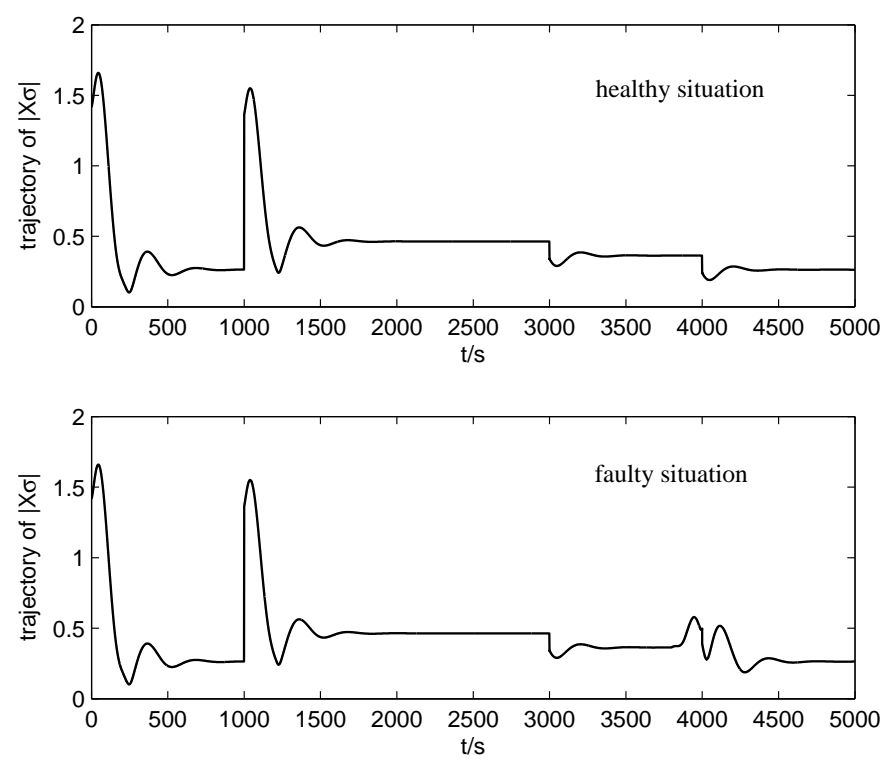

Figure 4: Trajectories of $\left|X_{\sigma(t)}(t)\right|$

To evaluate the individual controllers' performance, we illustrate the input norm trajectories of spacecraft 2 in $[0,4000) s$ until it leaves as shown in Fig. 5 where the system is healthy in $[0,3800) s$ and faulty after $t=3800 \mathrm{~s}$. We can see that the input converges to a small region in modes 1-3 since spacecraft 2 is not influenced by the formation change. After $t=3800 \mathrm{~s}$, the fault appears and makes the input magnitude become large. The input norm trajectories of other spacecraft are similar and thus are not shown here.

\section{Conclusion}

This paper investigates the spacecraft formation stabilization and fault tolerance from switched system point of view. Four phenomena of docking, undocking, departure and participation are incorporated with formation flying.

In the presence of obstacles that appear randomly, some obstacle avoidance actions have to be taken in the spacecraft level or the formation level [9]. This avoidance process can be regarded as 


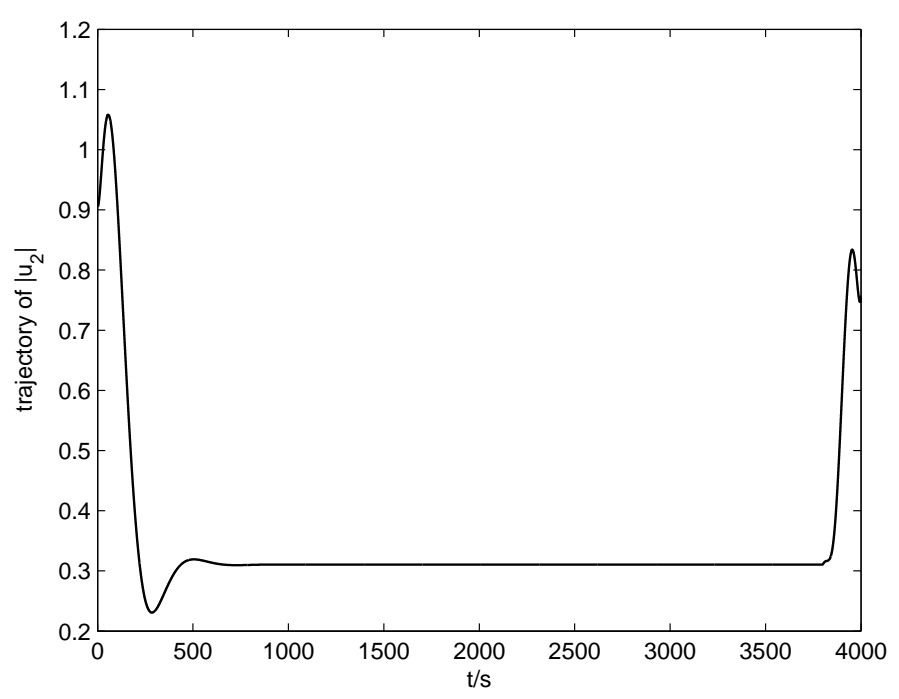

Figure 5: Trajectories of $\left|u_{2}(t)\right|$

a new mode of the original switched system, the proposed control architecture is potentially usable with some modifications of the switching law. This will be the focus of our future work.

\section{References}

[1] K. T. Alfriend, S. Vadali, P. Gurfil, J. How, and L. Breger, Spacecraft Formation Flying: Dynamics, Control and Navigation, Elsevier, 2010.

[2] R. W. Beard, J. Lawton, and F. Y. Hadaegh, A coordination architecture for spacecraft formation control, IEEE Transactions on Control Systems Technology, 9(6), 777-790, 2001.

[3] R. Kristiansen, P. J. Nicklasson, Spacecraft formation flying: a review and new results on state feedback control, Acta Astronautica, 65(11-12), 1537-1552, 2009.

[4] W. Ren, Formation keeping and attitude alignment for multiple spacecraft through Local Interactions, AIAA Journal of Guidance, Control and Dynamics, 30(2), 633-638, 2007.

[5] H. Du, S. Li and C. Qian, Finite-time attitude tracking control of spacecraft with application to attitude synchronization, IEEE Transactions on Automatic Control, 56(11), 2711-2717, 2011.

[6] H. Du, and S. Li, Finite-time cooperative attitude control of multiple spacecraft using terminal sliding mode control technique, International Journal of Modelling, Identification and Control, 16(4), 327-333, 2012. 
[7] H. Sun, S. Li, and S. Fei. A composite control scheme for 6DOF spacecraft formation control, Acta Astronautica, 69 (7-8), 595-611, 2011.

[8] H. Pan, and V. Kapila, Adaptive nonlinear control for spacecraft formation flying with coupled translational and attitude dynamics, Proceedings of the 40th IEEE Conference on Decision and Control, Orlando, Florida, 2001, 2057-2062.

[9] J. Walls, A. Howard, A. Homaifar, B. Kimiaghalam, A generalized framework for autonomous formation reconfiguration of multiple spacecraft, Proceedings of IEEE Aerospace Conference, 397-406, 2005.

[10] Hussein, D. Scheeres, A. Bloch, D. Hyland, and N. H. Mcclamroch, Optimal motion planning for dual-spacecraft interferometry, IEEE Transactions on Aerospace and Electronic Systems, 43(2), 723-737, 2007.

[11] M. Blanke, M. Kinnaert, J. Lunze, and M. Staroswiecki, Diagnosis and Fault-Tolerant Control, 2nd edition, Springer Verlag Berlin Heidelberg, 2006.

[12] Q. L. Hu, B. Xiao, and Y. M. Zhang, Fault-tolerant attitude control for spacecraft under loss of actuator effectiveness, AIAA Journal of Guidance, Control, and Dynamics, 34(3), 927-932, 2011.

[13] B. Xiao, Q. L. Hu, and Y. M. Zhang, Fault-tolerant attitude control for flexible spacecraft without angular velocity magnitude measurement, AIAA Journal of Guidance, Control, and Dynamics, 34(5), 1556-1561, 2011.

[14] A. Barua, and K. Khorasani, Hierarchical fault diagnosis and fuzzy rule-based reasoning for satellites formation flight, IEEE Transactions on Aerospace and Electronic Systems, 47(4), 2435-2456, 2011.

[15] S. M. Azizi, and K. Khorasani, A hierarchical architecture for cooperative fault accommodation of formation flying satellites in deep space, Proceedings of 2009 American Control Conference, 4178-4183, 2009.

[16] P. K. C. Wang, and F. Y. Hadaegh, Stability analysis of switched dynamical systems with state-space dilation and contraction, Proceedings of AIAA Guidance, Navigation, and Control Conference, Keystone, Colorado, AIAA 2006-6418, 2006.

[17] D. Liberzon, Switching in Systems and Control, Boston, MA: Birkhauser, 2003.

[18] J. Zhao, and D. J. Hill, On stability, $L_{2}$ gain and $H_{\infty}$ control for switched systems, Automatica, 44(5), 1220-1232, 2008. 
[19] P. Colaneri, J. C. Geromel, and A. Astolfi, Stabilization of continuous-time switched nonlinear systems, Systems \& Control Letters, 57(1), 95-103, 2008.

[20] H. Yang, M. Staroswiecki, B. Jiang, and J. Liu, Fault tolerant cooperative control for a class of nonlinear multi-agent systems, Systems 83 Control Letters, 60(4), 271-277, 2011.

[21] H. Yang, B. Jiang and V. Cocquempot, Fault Tolerant Control Design For Hybrid Systems, Springer-Verlag, Berlin Heidelberg, 2010.

[22] J. Hespanha, and A. S. Morse. Switching between stabilizing controllers, Automatica, 38(11), 19051917, 2002.

[23] R. Farshad, J. Hespanha, and G. Stewart, On controller initialization in multivariable switching systems, Automatica, 48(12), 3157-3165, 2012.

[24] W. Fehse, Automated Rendezvous and Docking of Spacecraft, Cambridge University Press, Cambridge, 2003.

[25] B. Wie, Space Vehicle Dynamics and Control (2nd Edition), AIAA, 2008. 